\title{
Acoustic Tests of Lorentz Symmetry Using Quartz Oscillators
}

\author{
Anthony Lo, Philipp Haslinger, Eli Mizrachi, Loïc Anderegg, and Holger Müller \\ Department of Physics, University of California, Berkeley, California 94720, USA
}

\author{
Michael Hohensee ${ }^{\dagger}$ \\ Lawrence Livermore National Laboratory, Livermore, California 94550, USA \\ Maxim Goryachev and Michael E. Tobar \\ ARC Centre of Excellence for Engineered Quantum Systems, School of Physics,
}

University of Western Australia, 35 Stirling Highway, Crawley, Western Australia 6009, Australia

(Received 8 December 2014; revised manuscript received 6 July 2015; published 24 February 2016)

\begin{abstract}
We propose and demonstrate a test of Lorentz symmetry based on new, compact, and reliable quartz oscillator technology. Violations of Lorentz invariance in the matter and photon sector of the standard model extension generate anisotropies in particles' inertial masses and the elastic constants of solids, giving rise to measurable anisotropies in the resonance frequencies of acoustic modes in solids. A first realization of such a "phonon-sector" test of Lorentz symmetry using room-temperature stresscompensated-cut crystals yields $120 \mathrm{~h}$ of data at a frequency resolution of $2.4 \times 10^{-15}$ and a limit of $\tilde{c}_{Q}^{n}=(-1.8 \pm 2.2) \times 10^{-14} \mathrm{GeV}$ on the most weakly constrained neutron-sector $c$ coefficient of the standard model extension. Future experiments with cryogenic oscillators promise significant improvements in accuracy, opening up the potential for improved limits on Lorentz violation in the neutron, proton, electron, and photon sector.
\end{abstract}

DOI: 10.1103/PhysRevX.6.011018

\author{
Subject Areas: Acoustics, \\ Atomic and Molecular Physics, \\ Electronics
}

\section{INTRODUCTION}

The possibility that physics beyond the standard model might violate Lorentz invariance [1-3] has motivated experimental tests with high precision and broad scope. In particular, experiments have placed stringent limits on anisotropies in the laws of motion of the photon, electron, proton, and neutron based on, e.g., electromagnetic cavities [4-7], clock comparisons [8-13], magnetometry [14-18], ultracold neutrons [19], and ion traps [20]. Some anisotropic inertial masses of particles are known to be below $10^{-28} \mathrm{GeV}$ [18], but others are more weakly constrained. Bounding all modes of Lorentz violation often requires active rotation and Earth's orbit to modulate the orientation and velocity of the apparatus, and thus data taking over a year [11,21-23], but at the same time uses fragile and maintenance-intensive atomic and optical setups. In this paper, we introduce the concept of an acoustic test of

\footnotetext{
*hm@berkeley.edu

${ }^{\dagger}$ Also at Department of Physics, University of California, Berkeley, CA 94720, USA.

Published by the American Physical Society under the terms of the Creative Commons Attribution 3.0 License. Further distribution of this work must maintain attribution to the author(s) and the published article's title, journal citation, and DOI.
}

Lorentz symmetry, based on precision measurements of phonon oscillations in a quartz crystal oscillator, demonstrating a simple and reliable, yet sensitive, method that is readily suited for long-term operation on a turntable or even being carried on small air and space vehicles. Based on commercial stress-compensated-cut quartz oscillators, we limit the most weakly constrained mode of neutron-sector violations in the standard model extension (SME) to $(-1.8 \pm 2.2) \times 10^{-14} \mathrm{GeV}$, improving on previous laboratory experiments [24] by 3 orders of magnitude and on a previous astrophysics bound by about 1 order of magnitude. This rules out all possibilities for Lorentz-violating anisotropies in the inertial mass of neutrons, protons, and electron at the $\sim 10^{-14} \mathrm{GeV}$ level. We show that future experiments with cryogenic oscillators could be used to perform more sensitive tests of Lorentz symmetry in the proton, neutron, electron, and photon sectors.

Our method is the first to compare acoustic oscillations in different directions to constrain Lorentz symmetry. In this work, we show that the frequencies of the bulk elastic waves are sensitive to the photon, electron, proton, and neutron sector of the SME. The coefficients of the SME that change the resonance frequency of acoustic modes in solids are collectively referred to as phonon-sector coefficients, in analogy to the photon sector. This work also paves the way for future experiments that involve high- $Q$ 
frequency stable phonon systems, which could be adapted to test Lorentz symmetry. Such systems include, but are not limited to, phonon lasers [25], optomechanical systems [26-28], and phonon-induced Brillouin scattering devices [29-33].

Hundreds of limits on Lorentz invariance violations do exist [34], but there are as many gaps where large signals may lie undetected. The gaps are typically left behind by other technologies because filling them would take rotating setups, long-term data taking, or operation in difficult environments. Our method is well suited for this task. We do note, however, that nonstandard phonon-sector signals may exist for reasons other than encoded in the SME, and this may lead to genuinely new tests of fundamental laws of physics.

\section{THEORY}

\section{A. Standard model extension}

Lorentz invariance violation has been parametrized in several ways; see e.g., Refs. [35-38]. We use a phenomenological framework known as the standard model extension [1-3] to describe the effects of Lorentz violation. It augments the standard model with new combinations of known particles and fields that lead to Lorentz violation, subject to the requirement that the theory must respect conservation of energy and momentum, renormalizability, gauge invariance, and observer Lorentz covariance. The new terms in the SME are parametrized by tensors, whose component values are collectively known as Lorentzviolation coefficients. If all such coefficients are zero, Lorentz symmetry is exact. The value of these Lorentzviolating coefficients is by definition frame dependent, but can be taken as approximately constant in any frame that is inertial on all time scales relevant to the experiment. It is conventional to use a Sun-centered celestial equatorial reference frame. Quantities in this frame are denoted by capital indices $T, X, Y, Z$. The time coordinate $T$ has its origin at the 2000 vernal equinox. The $Z$ axis is directed north and parallel to the rotational axis of Earth at $T=0$. The $X$ axis points from the Sun towards the vernal equinox, while the $Y$ axis completes a right-handed system [34].

\section{B. $c$ coefficients}

We study the influence of $c$-type coefficients in detail here. Later, we give an overview of all fermion- and photon-sector coefficients of the minimal SME and what levels of sensitivity can be expected for them in phononsector experiments.

The coefficients $c_{\mu \nu}$ enter the Lagrangian of a free Dirac fermion $w$ by substituting the Dirac matrix $\gamma \rightarrow \gamma_{\nu}+c_{\mu \nu} \gamma^{\mu}$, where $\nu=0,1,2,3$ are the space-time coordinates [8]. The term enters the nonrelativistic Schrödinger Hamiltonian of a particle by the substitution

$$
\frac{p^{2}}{2 m} \rightarrow \frac{p_{j} p_{k}}{2 m}\left(\delta_{j k}-2 c_{j k}^{w}-c_{00} \delta_{j k}\right),
$$

where $p_{j}$ are the components of momentum, $j, k=1,2,3$, and $m$ is the particle mass. Thus, the $c$ coefficients describe anisotropies of the inertial mass of particles that depend on the direction of its motion. (Though protons and neutrons are composite particles, they are approximated as Dirac fermions for the purpose of parametrizing Lorentz violation at low energies.) The electron, proton, and neutron tensors, $c_{\mu \nu}^{e}, c_{\mu \nu}^{p}$, and $c_{\mu \nu}^{n}$, are independent of one another, symmetric, and traceless with nine independent degrees of freedom each.

For a composite object $T$ that consists of $n^{w}$ particles of species $w$, the effects of Lorentz violation in Eq. (1) are given by effective coefficients

$$
c_{\mu \nu}^{T}=\frac{1}{m^{T}} \sum_{w} n^{w} m^{w} c_{\mu \nu}^{w}, \quad m^{T}=\sum_{w} n^{w} m^{w} .
$$

Not all of the basic $c_{\mu \nu}$ coefficients are physical. The physical combinations are conventionally expressed by the combinations [34]

$$
\begin{aligned}
\tilde{c}_{Q}^{w} & =m^{w}\left(c_{X X}^{w}+c_{Y Y}^{w}-2 c_{Z Z}^{w}\right), \\
\tilde{c}_{-}^{w} & =m^{w}\left(c_{X X}-c_{Y Y}\right), \\
\tilde{c}_{J}^{w} & =m^{w}\left|\varepsilon_{J K L}\right| c_{K L}, \\
\tilde{c}_{T J}^{w} & =m^{w}\left(c_{T J}+c_{J T}\right), \\
\tilde{c}_{T T}^{w} & =m^{w} c_{T T} .
\end{aligned}
$$

We use these combinations throughout when stating experimental results. We note that by these definitions, $\tilde{c}_{T J} \neq m c_{T J}$.

\section{Relative significance of the terms}

The components of $\tilde{c}$ encode independent degrees of freedom for Lorentz violation. Knowledge of one or many of them does not imply anything about the remaining ones. By analogy, in the photon sector several different modes of Lorentz violation exist, which are characterized by how they transform under Lorentz boosts and rotations. Some of them, the $\tilde{\kappa}_{e+}$ and $\tilde{\kappa}_{o-}$, have been bounded astrophysically to an accuracy of $10^{-34}$ [39]. Other coefficients (denoted $\tilde{\kappa}_{e-}$ and $\tilde{\kappa}_{o+}$ ) remain unexplored by these types of observations. They are best studied by laboratory experiments, which have been continuously improved from the original ones by Michelson and Morley, Kennedy and Thorndike, and Ives and Stilwell [40] to modern ones that reach down to sensitivities of $10^{-18}[4-6,41,42]$. But even those tests leave behind a last remaining coefficient. This one, $\tilde{\kappa}_{\text {tr }}$, is arguably the hardest to measure, as dedicated experiments were set up to measure this remaining coefficient, and now 
all modes of Lorentz violation in the minimal photon sector have been very stringently limited by experiment $[5,43,44]$.

By comparison, the fermion sectors have been studied less comprehensively. Here, also, some components of the coefficients for Lorentz violation have been limited with high precision, but others remain tested at low precision.

\section{Influence of the fermion $c$ coefficients in crystal oscillators}

The principle of our search for anisotropic inertial masses is simple: We use a quartz oscillator performing nominally $10-\mathrm{MHz}$ oscillations on a turntable. If the inertial mass of the quartz material in one direction is fractionally higher by $\delta m / m$ than in an orthogonal direction, then rotating the crystal leads to a modulation of the oscillation frequency by $(\delta \nu / \nu)=-\frac{1}{2} \delta m / m$. We use shear oscillations in a SC-cut quartz crystal. This modulation can be measured, either by comparison to a stationary reference or by comparison to a second oscillator on the turntable, rotated by $90^{\circ}$ relative to the first.

Finding the sensitivity of mechanical resonators to Lorentz violation is possible by perturbation theory for each eigenmode. Our experiment uses a stress compensated cut [45] crystal bulk acoustic wave (BAW) piezoelectric plate resonator working at the third overtone of the thickness shear mode. This resonator is housed in an oven at the temperature of around $85^{\circ} \mathrm{C}$, where the vibrational mode exhibits zero temperature coefficient of its oscillation frequency. The SC cut is doubly rotated relative to the crystal axis by a first angle of $\theta=34.11^{\circ}$ and a second angle $\phi=21.93^{\circ}$. This also results in zero stress dependence of the frequency, which reduces the dependence of the frequency on the mounting of the crystal, amplitude variations of the oscillation, and aging [46].

\section{Unperturbed modes}

The eigenmodes of doubly rotated piezoelectric plate resonators have been studied in detail $[47,48]$. Because of high $Q$ factors (typically slightly above $10^{6}$ at room temperature) the eigenmodes may be considered isolated mechanical oscillators. We introduce a plate coordinate system $x^{[i]}(i=1,2,3)$ in which $x^{[2]}$ is normal to the major surfaces of the doubly rotated quartz blank, $x^{[1]}$ is directed along the axis of the second rotation, and $x^{[3]}$ is completing a right-handed system (Table I). We denote $u^{[i]}(t, x)$ the components of the displacement of a volume element at $x^{[i]}$ as a function of time $t$. We start by finding the modes that depend only on the $x^{[2]}$ coordinate ("thickness modes"),

$$
u^{[r]}=A^{[r]} \sin \left(\eta x^{[2]}\right) e^{i \omega t},
$$

where

$$
\left(\overline{\hat{c}}_{[2 n r 2]}-\bar{c} \delta_{[n r]}\right) A^{[r]}=0 .
$$

\begin{tabular}{|c|c|c|}
\hline Name & Notation & Description \\
\hline \multirow[t]{3}{*}{ Blank } & $x^{[1]}$ & $\begin{array}{l}\text { Axis of second crystal rotation; } \\
\text { approximately direction of shear }\end{array}$ \\
\hline & $x^{[2]}$ & Normal to major blank surface \\
\hline & $x^{[3]}$ & Completes right-handed system \\
\hline Thickness & $x^{(i)}$ & Parallel to thickness modes \\
\hline \multirow[t]{3}{*}{$\mathrm{Lab}$} & $x^{1}=x$ & Horizontally pointing south \\
\hline & $x^{2}=y$ & Horizontally pointing east \\
\hline & $x^{3}=z$ & Vertically upwards \\
\hline \multirow[t]{4}{*}{ Sun-centered } & $x^{T}=T$ & $T=0$ at 2000 vernal equinox \\
\hline & $x^{X}$ & From Sun towards vernal equinox \\
\hline & $x^{Y}$ & Completes right-handed system \\
\hline & $x^{Z}$ & Parallel to Earth's axis pointing north \\
\hline
\end{tabular}

TABLE I. Coordinates used in this paper.

The $\bar{c}_{[2 n r 2]}$ are the piezoelectrically stiffened elastic constants rotated into the blank coordinate system. Solving the last equation yields three eigenvectors $\left(A^{(1-3)}\right)^{[r]}$. These eigenvectors are used as the basis of new "thickness" coordinates $x^{(1-3)}$, organized such that $x^{(i)}$ has its largest component along $x^{[i]}$. Analysis in the thickness mode coordinates then yields three mode families, known as quasilongitudinal $(A)$ mode, fast shear $(B)$ mode, and slow shear $(C)$ mode. For each family, the amplitude of one of the displacement components in $x^{(i)}$ direction is large while the others are small. Because of this smallness, the modes nearly decouple, which makes it possible to find accurate closed-form expressions for the eigenmodes.

The modes of interest here have the largest displacement component along $x^{(1)}$, which is approximately along the $x^{[1]}$ axis. It can be written as [48]

$u_{1 n m p}=e^{-\alpha_{1 n} x_{1}^{2} / 2} H_{m}\left(\sqrt{\alpha_{1 n}} x_{1}\right) e^{-\beta_{1 n} x_{3}^{2} / 2} H_{p}\left(\sqrt{\beta_{1 n}} x_{3}\right)$,

where

$$
\alpha_{1 n}^{2}=\frac{n^{2} \pi^{2} \hat{c}^{(1)}}{8 R h_{0}^{3} M_{1 n}^{\prime}}, \quad \beta_{1 n}^{2}=\frac{n^{2} \pi^{2} \hat{c}^{(1)}}{8 R h_{0}^{3} P_{1 n}^{\prime}},
$$

and $H_{m}$ is the Hermite polynomial of order $m$. For the mode used in a third-overtone SC-cut crystal at $10 \mathrm{MHz}, n=3$, $m=p=0, M_{1 n}^{\prime}=5.3273, P_{1 n}^{\prime}=6.3858, \hat{c}^{(1)}=3.4379$, $R$ is the radius of the blank, and $h_{0}$ is the thickness, which is $0.54094 \mathrm{~mm}$ to make the resonance frequency $10 \mathrm{MHz}$ [48].

\section{Perturbation due to Lorentz violation}

Since the motion of the volume elements is primarily in the $x^{[1]}$ direction, Eq. (1) predicts that

$$
\frac{p_{[x]}^{2}}{2 m} \rightarrow \frac{p_{[x]}^{2}}{2 m}\left(1-2 c_{[x x]}-c_{[00]}\right),
$$


which is equivalent to a rescaling of the inertial mass by $1+2 c_{[x x]}+c_{00}$. Solids are composite objects; summing up the contributions of the rescalings in the electron, proton, and neutron sectors amounts to replacing the coefficients by the effective coefficients Eq. (2). This leads to a relative change in the resonance frequency of

$$
\frac{\delta \nu}{\nu}=-\frac{1}{2}\left(2 c_{[x x]}^{T}+c_{00}^{T}\right) .
$$

To study a simple case first, we may assume that all coefficients of Eq. (3) are zero except for $\tilde{c}_{Q}$, that the experiment with two rotating quartz oscillators is located at the equator with the $[x]$ axis horizontal and rotated around a vertical axis at an angular velocity of $\omega_{t}$. This would lead to a modulation amplitude of $\delta \nu / \nu=c_{Q}^{Q} / 4$, where the superscript $Q$ indicates we are using the effective combination of coefficients for quartz.

For the general case, we calculate the components $c_{[x]}$ in the crystal frame, rotating on the turntable, from the $c_{\mu \nu}$ in the Sun-centered frame. This involves Lorentz boosts and rotations [8]. We denote $\omega_{t}$ the angular velocity of the turntable measured in the lab frame, $\omega_{\oplus} \approx 2 \pi /(23 h 56 \mathrm{~min})$, and $\Omega_{\oplus}=2 \pi /(1 \mathrm{y})$ the sidereal angular velocities of Earth's rotation and orbit, respectively, $\chi$ is the colatitude of the laboratory in which the experiment is performed $\left(\chi \approx 52.13^{\circ}\right.$ for the current experiment in Berkeley, California), and $\eta \approx 23.4^{\circ}$ is the angle between the ecliptic and Earth's equatorial plane.

The signal either includes contributions of order 1 , or is suppressed by the Earth's orbital velocity $\beta_{\oplus} \approx 10^{-4}$. We neglect contributions from signals suppressed by higher powers of $\beta_{\oplus}$ or by the velocity of the laboratory due to Earth's rotation $\beta_{L} \approx 10^{-6}$. We express the measured frequency variation as a Fourier series,

$$
\frac{\delta \nu}{\nu}=\frac{1}{8} \sum_{l, m, n}\left(C_{l m n} \cos \omega_{l m n} T+S_{l m n} \sin \omega_{l n m} T\right),
$$

where the factor of $1 / 8$ is to simplify the Fourier coefficients $C_{l m n}, S_{l m n}$, and

$$
\omega_{l m n}=l \omega_{t}+m \omega_{\oplus}+n \Omega_{\oplus}
$$

The Fourier coefficients are listed in Tables II and III. For the purpose of these tables, we use the definitions

TABLE II. Signal components for one rotating crystal oscillator compared against a stationary reference that is not affected by the $c$

\begin{tabular}{|c|c|c|}
\hline$\underline{l, m, n}$ & $\cos$ & $\sin$ \\
\hline dc & $-2 c_{Q}^{T}\left(\sin ^{2} \chi-2\right)$ & \\
\hline $0,0,1$ & $2\left[-2 c_{T Z}^{T} \sin \eta \sin ^{2} \chi+\cos \eta c_{T Y}^{T}\left(\sin ^{2} \chi-2\right)\right] \beta_{\oplus}$ & $2\left(1+\cos ^{2} \chi\right) c_{T X}^{T} \beta_{\oplus}$ \\
\hline $0,1,-1$ & $2 \cos \chi \sin \eta \beta_{\oplus} c_{T X}^{T}$ & $2 \cos \chi\left[c_{T Z}^{T}(1+\cos \eta)+c_{T Y}^{T} \sin \eta\right] \sin \chi \beta_{\oplus}$ \\
\hline $0,1,0$ & $-2 \sin \chi\left(2 \cos \chi c_{Y}^{T}+c_{-}^{T} \sin \chi\right)$ & $-4 \cos \chi \sin \chi c_{X}^{T}$ \\
\hline $0,1,1$ & $2 \cos \chi c_{T X}^{T} \sin \eta \sin \chi$ & $2 \cos \chi\left[(\cos \eta-1) c_{T Z}^{T}+c_{T Y}^{T} \sin \eta\right] \sin \chi \beta_{\oplus}$ \\
\hline $0,2,-1$ & $(1+\cos \eta)\left(\cos ^{2} \chi-1\right) \beta_{\oplus} c_{T Y}^{T}$ & $-(1+\cos \eta)\left(\cos ^{2} \chi-1\right) c_{T X}^{T} \beta_{\oplus}$ \\
\hline $0,2,0$ & & $2\left(\cos ^{2} \chi-1\right) c_{Z}^{T}$ \\
\hline $0,2,1$ & $(\cos \eta-1)\left(\cos ^{2} \chi-1\right) c_{T Y}^{T} \beta_{\oplus}$ & $-(\cos \eta-1)\left(\cos ^{2} \chi-1\right) c_{T X}^{T} \beta_{\oplus}$ \\
\hline $2,-2,-1$ & $(\cos \eta-1)(1-\cos \chi)^{2} c_{T Y}^{T} \beta_{\oplus} / 2$ & $(\cos \eta-1)(\cos \chi-1)^{2} c_{T X}^{T} \beta_{\oplus} / 2$ \\
\hline $2,-2,0$ & $c_{-}^{T}\left(2-2 \cos \chi-\sin ^{2} \chi\right)+2(1-\cos \chi) c_{Y}^{T} \sin \chi$ & $-(\cos \chi-1)^{2} c_{Z}^{T}$ \\
\hline $2,-2,1$ & $(1+\cos \eta)(1-\cos \chi)^{2} c_{T Y}^{T} \beta_{\oplus} / 2$ & $(1+\cos \eta)(\cos \chi-1)^{2} c_{T X}^{T} \beta_{\oplus} / 2$ \\
\hline $2,-1,-1$ & $(\cos \chi-1) \sin \eta \sin \chi c_{T X}^{T} \beta_{\oplus}$ & $-(\cos \chi-1)\left[(\cos \eta-1) c_{T Z}^{T}+\sin \eta c_{T Y}^{T}\right] \sin \chi \beta_{\oplus}$ \\
\hline $2,-1,0$ & & $2(\cos \chi-1) c_{X}^{T}$ \\
\hline $2,-1,1$ & $(\cos \chi-1) c_{T X}^{T} \sin \eta \beta_{\oplus}$ & $-(\cos \chi-1)\left[c_{T Z}^{T}(1+\cos \eta)+c_{T Y}^{T} \sin \eta\right] \sin \chi \beta_{\oplus}$ \\
\hline $2,0,-1$ & $\left(\cos \eta c_{T Y}^{T}-2 c_{T Z}^{T} \sin \eta\right) \sin ^{2} \chi \beta_{\oplus}$ & $-\left(-1+\cos ^{2} \chi\right) c_{T X}^{T} \beta_{\oplus}$ \\
\hline $2,0,0$ & $-2 c_{Q}^{T} \sin ^{2} \chi$ & \\
\hline $2,0,1$ & $-\left[\cos \eta\left(\cos ^{2} \chi-1\right) c_{T Y}^{T}+2 c_{T Z}^{T} \sin \eta \sin ^{2} \chi\right] \beta_{\oplus}$ & $\left(\cos ^{2} \chi-1\right) c_{T X}^{T} \beta_{\oplus}$ \\
\hline $2,1,-1$ & $(1+\cos \chi) \sin \eta \sin \chi \beta_{\oplus} c_{T X}^{T}$ & $(1+\cos \chi)\left[c_{T Z}^{T}(1+\cos \eta)+c_{T Y}^{T} \sin \eta\right] \sin \chi \beta_{\oplus}$ \\
\hline $2,1,0$ & $-2(1+\cos \chi) \sin \chi c_{Y}^{T}$ & $-2(1+\cos \chi) c_{X}^{T}$ \\
\hline $2,1,1$ & $(1+\cos \chi) c_{T X}^{T} \sin \eta \beta_{\oplus}$ & $(1+\cos \chi)\left[(\cos \eta-1) c_{T Z}^{T}+c_{T Y}^{T} \sin \eta\right] \sin \chi \beta_{\oplus}$ \\
\hline $2,2,-1$ & $(1+\cos \eta)(1+\cos \chi)^{2} \beta_{\oplus} c_{T Y}^{T} / 2$ & $-(1+\cos \eta)(1+\cos \chi)^{2} c_{T X}^{T} \beta_{\oplus} / 2$ \\
\hline $2,2,0$ & $c_{-}^{T}\left(2+2 \cos \chi-\sin ^{2} \chi\right)$ & $(1+\cos \chi)^{2} c_{Z}^{T}$ \\
\hline $2,2,1$ & $(\cos \eta-1)(1+\cos \chi)^{2} c_{T Y}^{T} \beta_{\oplus} / 2$ & $-(\cos \eta-1)(1+\cos \chi)^{2} c_{T X}^{T} \beta_{\oplus} / 2$ \\
\hline
\end{tabular}
coefficients. Signal components suppressed by $\beta_{\oplus}^{2}$ and higher powers are omitted. These coefficients are to be inserted in Eq. (9) and are multiplied by $1 / 8$ to give the frequency change. 
TABLE III. Signal components for an experiment with two rotating crystal oscillators compared against one another. Components suppressed by $\beta_{\oplus}^{2}$ and higher powers have been omitted. These coefficients are to be inserted in Eq. (9) and are multiplied by $1 / 8$ to give the frequency change.

\begin{tabular}{|c|c|c|}
\hline$l, m, n$ & $\cos$ & $\sin$ \\
\hline $\mathrm{dc}$ & $-4 c_{O}^{T} \cos (2 \theta)$ & \\
\hline $2,-2,-1$ & $(\cos \eta-1)(\cos \chi-1)^{2} c_{T Y}^{T} \beta_{\oplus}$ & $(\cos \eta-1)(\cos \chi-1)^{2} c_{T X}^{T} \beta_{\oplus}$ \\
\hline $2,-2,0$ & $2(\cos \chi-1)^{2} c_{M}^{T}$ & 0 \\
\hline $2,-2,1$ & $(1+\cos \eta)(-1+\cos \chi)^{2} c_{T Y}^{T} \beta_{\oplus}$ & $(1+\cos \eta)(\cos \chi-1)^{2} c_{T X}^{T} \beta_{\oplus}$ \\
\hline $2,-1,-1$ & $2(\cos \chi-1) c_{T X}^{T} \sin \eta \sin \chi \beta_{\oplus}$ & $2(1-\cos \chi)\left[(\cos \eta-1) c_{T Z}^{T}+c_{T Y}^{T} \sin \eta\right] \sin \chi \beta_{\oplus}$ \\
\hline $2,-1,0$ & $4(\cos \chi-1) c_{Y}^{T} \sin \chi$ & $4(\cos \chi-1) c_{X}^{T} \sin \chi$ \\
\hline $2,-1,1$ & $2(\cos \chi-1) c_{T X}^{T} \sin \eta \sin \chi \beta_{\oplus}$ & $2(1-\cos \chi)\left(c_{T Z}^{T}+\cos \eta c_{T Z}^{T}+c_{T Y}^{T} \sin \eta\right) \sin \chi \beta_{\oplus}$ \\
\hline $2,0,-1$ & $2 \sin ^{2} \chi\left(\cos \eta c_{T Y}^{T}-2 c_{T Z}^{T} \sin \eta\right) \beta_{\oplus}$ & $2 \sin ^{2} \chi c_{T X}^{T} \beta_{\oplus}$ \\
\hline $2,0,0$ & $-4 \sin ^{2} \chi c_{Q}^{T}$ & 0 \\
\hline $2,0,1$ & $2 \sin ^{2} \chi\left(\cos \eta c_{T Y}^{T}-2 c_{T Z}^{T} \sin \eta\right) \beta_{\oplus}$ & $-2 \sin ^{2} \chi c_{T X}^{T} \beta_{\oplus}$ \\
\hline $2,1,-1$ & $2(1+\cos \chi) c_{T X}^{T} \sin \eta \sin \chi \beta_{\oplus}$ & $2(1+\cos \chi)\left(c_{T Z}^{T}+\cos \eta c_{T Z}^{T}+c_{T Y}^{T} \sin \eta\right) \sin \chi \beta_{\oplus}$ \\
\hline $2,1,0$ & $-4(1+\cos \chi) c_{Y}^{T} \sin \chi$ & $-4(1+\cos \chi) c_{X}^{T} \sin \chi$ \\
\hline $2,1,1$ & $2(1+\cos \chi) c_{T X}^{T} \sin \eta \sin \chi \beta_{\oplus}$ & $2(1+\cos \chi)\left[(-1+\cos \eta) c_{T Z}^{T}+c_{T Y}^{T} \sin \eta\right] \sin \chi \beta_{\oplus}$ \\
\hline $2,2,-1$ & $(1+\cos \eta)(1+\cos \chi)^{2} c_{T Y}^{T} \beta_{\oplus}$ & $-(1+\cos \eta)(1+\cos \chi)^{2} c_{T X}^{T} \beta_{\oplus}$ \\
\hline $2,2,0$ & $2 c_{-}^{T}(1+\cos \chi)^{2}$ & $2(1+\cos \chi)^{2} c_{Z}^{T}$ \\
\hline $2,2,1$ & $(\cos \eta-1)(1+\cos \chi)^{2} c_{T Y}^{T} \beta_{\oplus}$ & $(1-\cos \eta)(1+\cos \chi)^{2} c_{T X}^{T} \beta_{\oplus}$ \\
\hline
\end{tabular}

$$
\begin{aligned}
c_{Q}^{T} & =c_{X X}^{T}+c_{Y Y}^{T}-2 c_{Z Z}^{T}, \\
c_{-}^{T} & =c_{X X}^{T}-c_{Y Y}^{T}, \quad c_{J}^{w}=\left|\varepsilon_{J K L}\right| c_{K L}^{T}, \\
c_{T J}^{T} & =c_{T J}^{T}+c_{J T}^{T}, \quad c_{T T}^{T}=c_{T T}^{T},
\end{aligned}
$$

similar to Eq. (3) but without the factor of particle mass. They are related to the physical, single-particle coefficients $\tilde{c}$ by, e.g., $c_{Q}^{T}=\sum_{w} \eta^{w} \tilde{c}_{Q}^{w}$, and so on. In this equation, $\eta^{w}=n^{w} / m^{T}$ is the number of particles of species $w$ per mass of the composite object. For naturally abundant quartz, the numbers of electrons, protons, and neutrons are quite similar and $\eta^{e} \simeq \eta^{p} \simeq \eta^{n} \simeq 0.53 / \mathrm{GeV}$.

\section{PRELIMINARY EXPERIMENT WITH ROOM-TEMPERATURE OSCILLATORS}

Optimized phonon-sector experiments will be able to improve bounds in all fermion sectors of the SME, as we see below. For our current experiment, however, we focus on the neutron sector. Existing limits on the proton- and electron-sector coefficients are at levels somewhat below the sensitivity of this preliminary experiment [34].

\section{A. Previous neutron-sector limits}

The most sensitive experiments to determine limits on the $\tilde{c}^{n}$ for neutrons are based on magnetometry [8,15,18,49-51]. In particular, a neon-rubidium-potassium comagnetometer has been used, which simultaneously senses the influence of background magnetic fields and the signal for Lorentz violation. This bounds the four spatial components $\tilde{c}_{J}^{n}, \tilde{c}$ to the very low level of $10^{-29}$ [18]. Limits on the fifth, $c_{Q}^{n}$, are not available from this experiment, but are available from tests of the weak equivalence principle [24] and astrophysics [52]. Without making untested assumptions about the character or degree to which Lorentz symmetry is broken in other sectors of the SME, the best laboratory limit on $c_{Q}^{n}$ is $\left|\tilde{c}_{Q}^{n}\right|<$ $10^{-8} \mathrm{GeV}$ [24]. Assuming that the $\alpha a_{\text {eff }}$ coefficients vanish, an improved limit of $10^{-11} \mathrm{GeV}$ is possible [24]. Astrophysics studies of the stability of cosmic-ray protons yields $\left|\tilde{c}_{Q}\right|<2 \times 10^{-13} \mathrm{GeV}$ and $\left|\tilde{c}_{T J}\right|<5 \times 10^{-14} \mathrm{GeV}$ [52]. The temporal $\tilde{c}_{T T}^{n}$ have been measured in atom interferometry [53].

\section{B. Setup}

Our experiment (Fig. 1) uses active rotation at a frequency of $\omega_{t}=2 \pi \times 0.36 \mathrm{~Hz}$ on a precision air-bearing turntable. Relative to experiments based solely on Earth's rotation, this increases the signal frequencies and thus allows us to suppress the drift of the oscillators due, e.g., to aging or temperature instability. The turntable (Professional Instruments, model 10R-606) is specified to $0.1 \mu \mathrm{rad}$ tilt of the rotation axis and $<25 \mathrm{~nm}$ radial and axial wobble, and has a specified stiffness of $10 \mathrm{Nm} / \mu \mathrm{rad}$. We use two oscillators that are rotating on the turntable and that are directly compared on the turntable. This avoids the need to bring the signals in or out of the turntable. (The target accuracy of $10^{-13}$ out of $10 \mathrm{MHz}$ requires us to detect phase modulations of microradian size; any modulations introduced when transmitting the signal from the turntable to the stationary laboratory frame would be synchronized with the putative signal, and none of the available methods can be trusted to not introduce tiny phase or amplitude modulations.) The oscillators (Stanford Research 
Systems SC-10) are signal generators classified as ovenized voltage-controlled crystal oscillators based on quartz SCcut BAW resonators. The oscillators are specified to an Allan variance of $2 \times 10^{-12}$ at $1 \mathrm{~s}$ averaging time. All components are highly reliable and covered with a mumetal shield, allowing the experiment to take uninterrupted data over long stretches of time.

Directly comparing the frequency of the oscillators via an available frequency counter is limited to a resolution of about $10^{-11}$ in $1 \mathrm{~s}$ by the $\sim 100$-ps timing resolution of the device. Much higher resolution can be achieved by using a double-balanced mixer (Mini-Circuits RPD-1) to measure the phase difference between the oscillators. We use the mixer's internal signal transformers to provide galvanic isolation between the quartz oscillators and the dc circuits (the RPD-1 allows the three ports to have separate grounds) to avoid dc signal errors through ground loops, given the large supply current of the quartz ovens. The output signal of the mixer is preamplified 1000 times and the resulting voltage $U$ is digitized on the turntable. The digital signal is brought out of the turntable via a universal serial bus

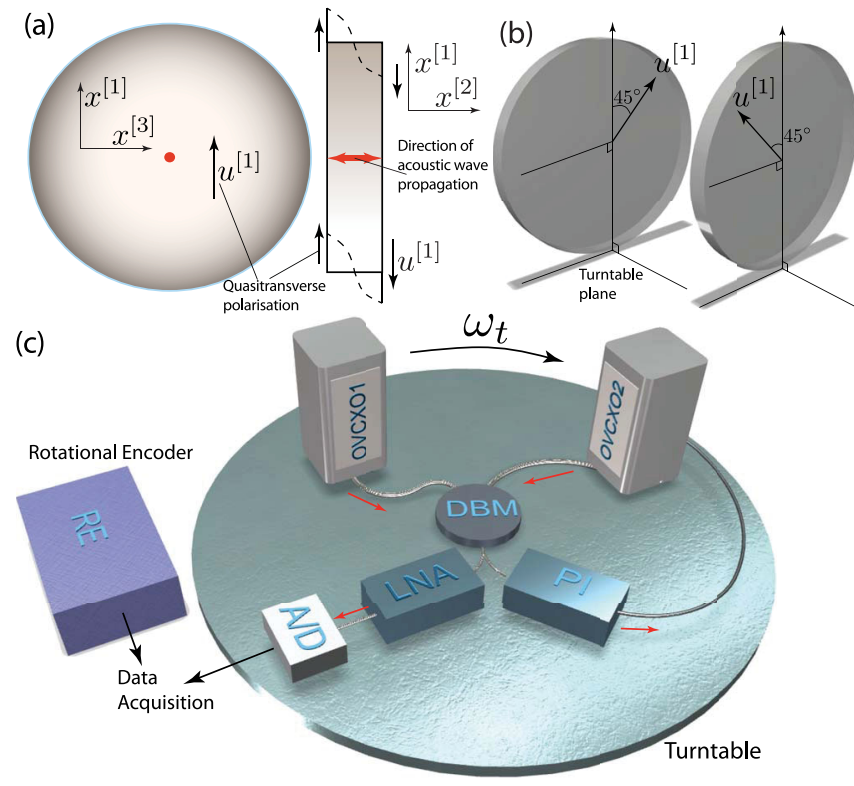

FIG. 1. Schematic of the room-temperature experiment. (a) Crystal blank showing coordinates $x^{[1-3]}$, the direction of propagation, and the direction of the displacement of the shear mode. (b) Orientation of a shear relative to the turntable plane. (c) Frequency comparison on the turntable: The phase between two $10-\mathrm{MHz}$ ovenized, voltage-controlled crystal oscillators (OVCXO) is detected by homodyne detection using a doublebalanced mixer (DBM). Phase lock with a PI feedback controller keeps the DBM operating near zero output voltage. The feedback is very slow, leaving the oscillators essentially free-running on the time scale of the turntable rotation rate and its harmonics. The DBM output is amplified by a low noise amplifier (LNA) and acquired after analog-to-digital (A/D) conversion. All components are enclosed in a cylindrical dual-layer mu-metal magnetic shield (not shown). connection through slip-ring contacts. Power at $15 \mathrm{~V}$ is also supplied via slip rings.

On time scales much longer than the rotation period of our turntable, we phase lock the oscillators together so that the mixer may always operate close to $90^{\circ}$ phase difference, i.e., near-zero output signal. The effective frequency-tovoltage conversion factor measured at the mixer output is thus zero at extremely low frequencies, where any voltages are removed by the feedback loop; at high frequencies, where the feedback is ineffective, the factor is given purely by the mixer itself. We measure the conversion efficiency of the mixer as a frequency discriminator by replacing one of the quartz oscillators with a digital synthesizer that provides a known frequency modulation. Figure 2 shows the measured response function. At our signal frequency of $2 \omega_{t} \sim 2 \pi \times 0.76 \mathrm{~Hz}$, we obtain $\delta \nu=\Delta U /(1.3 \mathrm{~V} / \mathrm{Hz})$.

The turntable is driven by an unregulated dc motor. Even small changes of the rotation rate accumulate to a large angle offset over time. We, therefore, use a light gate as a rotation encoder that delivers one pulse per turn to the computer, resetting the angle scale of the turntable rotation. The computer then interpolates linearly assuming a constant rotation rate during one turn.

\section{Results}

The system proves to be extremely reliable and capable of unattended operation. Figure 3 shows the amplitude Fourier transform of $120.0 \mathrm{~h}$ of data (about 164000 turntable rotations). Zooming into the region close to the expected signals around $2 \omega_{t}$ (Fig. 4) reveals sine and cosine amplitudes that are normally distributed with a standard deviation of $\sigma^{2}=\left\langle A_{c}^{2}\right\rangle=32 \mu \mathrm{V}$ after amplification. The measured signal at $2 \omega_{t}$ is $-26 \mu \mathrm{V}$. This corresponds to $(-26 \pm 32) \mathrm{nV}$ at the mixer output and, thus, $\delta \nu / \nu=$ $(-2.0 \pm 2.4) \times 10^{-15}$; see Fig. 2 .

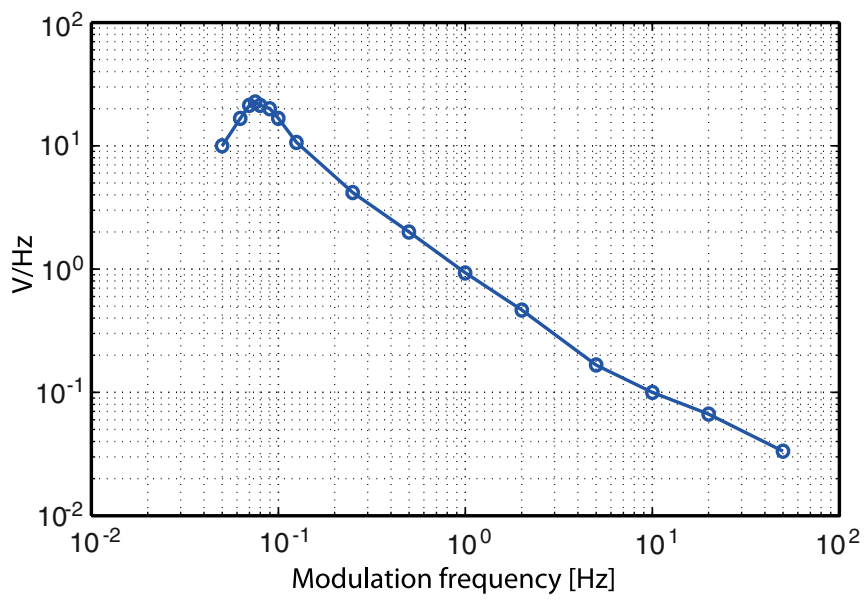

FIG. 2. Conversion efficiency of the mixer as frequency discriminator, measured with the phase-lock loop closed by inserting a signal having a known frequency modulation. 


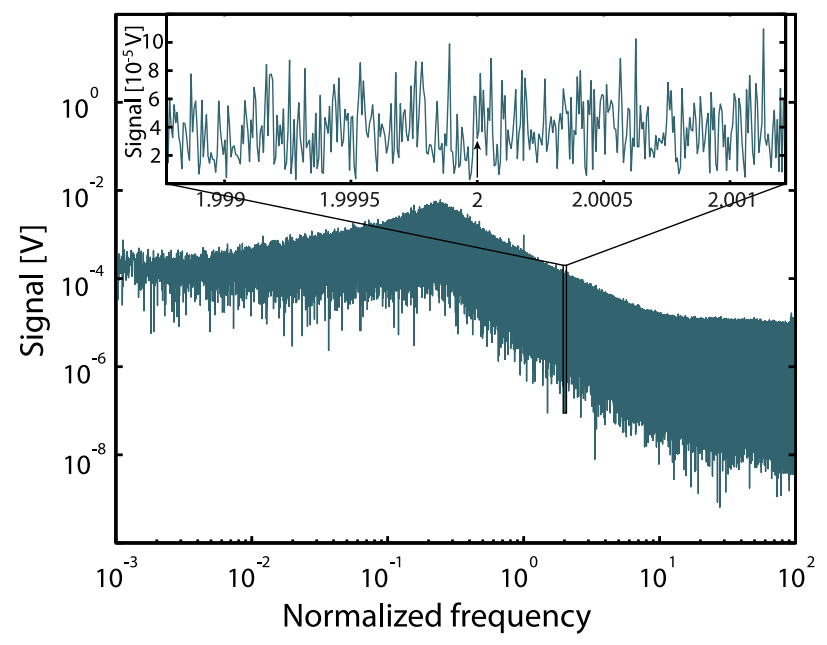

FIG. 3. Fourier transform of $120 \mathrm{~h}$ of data. Frequency is measured in multiples of the turntable rotation frequency. The inset shows the region around the expected signals.

The signal for Lorentz violation (Table III) has components at various frequencies around $2 \omega_{t}$. At our present accuracy, we restrict our analysis to the effect of $c_{Q}^{Q}$, which causes a signal proportional to $\cos \left(2 \omega_{t} T\right)$. For its amplitude, we find $\delta \nu / \nu=\frac{1}{2} \sin ^{2} \chi c_{Q}^{Q} \simeq 0.31 c_{Q}^{Q}$. In the experiment, however, the axes of the oscillators are oriented $45^{\circ}$ relative to the rotation axis, which we take into account by a factor of $\sin 45^{\circ}$. We thus find $c_{Q}^{Q}=$ $(-0.9 \pm 1.1) \times 10^{-14}$ on the effective coefficient for naturally abundant quartz, which translates into a limit of $\tilde{c}_{Q}^{n}=(-1.8 \pm 2.2) \times 10^{-14} \mathrm{GeV}$ on the neutron-sector coefficient.

Systematic effects of quartz oscillators, such as aging, temperature fluctuations and thermal hysteresis, acceleration, magnetic fields, power supply voltage, load impedance, electric fields, ionizing radiation, and ground loops,
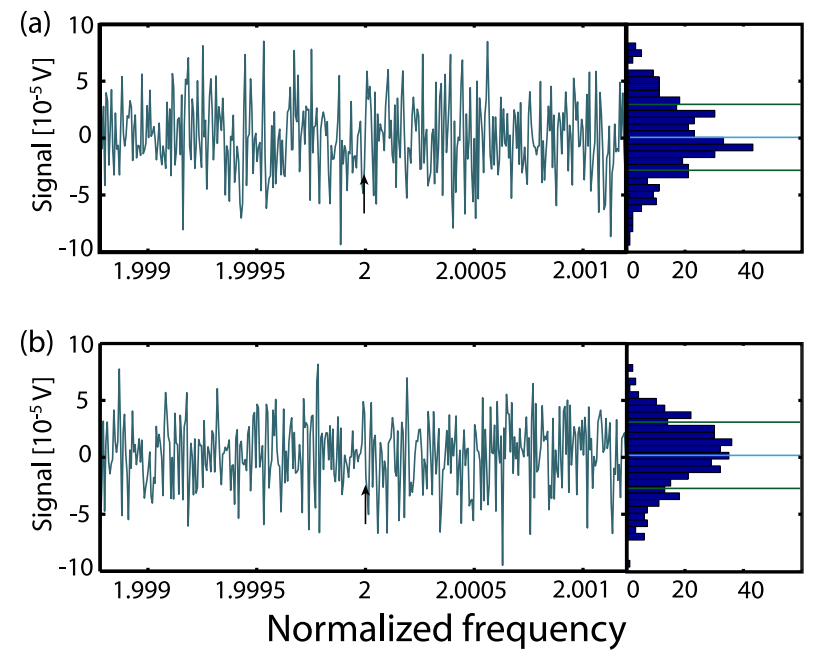

FIG. 4. Cosine (a) and sine (b) Fourier transform around $2 \omega_{t}$. The arrow points out the putative signal at $2 \omega_{t}$. are well understood. At our current resolution, most systematics are negligible, so here we discuss the largest two effects: We measure the acceleration sensitivity of our quartz oscillators by inverting them relative to Earth's gravitational acceleration $g$. For the most sensitive axis, we find $\delta \nu \sim 20 \mathrm{mHz} / 2 g$. The turntable wobble is specified to be less than $25 \mathrm{~nm}$ radially and axially. If we conservatively assume that this wobble contributes a $2 \omega_{t}$ frequency component (in reality, the energy of the wobble is likely spread out over many Fourier components), the corresponding acceleration is $25 \mathrm{~nm} \times 4 \omega_{t}^{2} \sim 0.14 \times 10^{-6} \mathrm{~g}$, which produces frequency changes of $2.8 \mathrm{nHz}$. Changing magnetic fields induce voltages into our wiring. Assuming $1 \mathrm{G}$ and an enclosed area of $1 \mathrm{~cm}^{2}$ at the turntable frequency $2 \omega_{t}$ (conservatively assuming that all the magnetic field will contribute to the second harmonic of the turn table rate), we obtain an induced voltage of $\sim 40 \mathrm{nV}$, comparable to our signal size. For this reason, we enclose the entire setup up to and including the amplifier in a two-layer mu-metal shield, which should reduce this influence at least $\sim 100$-fold.

\section{Cryogenic experiment}

The quartz bulk acoustic wave technology provides the most stable oscillators in the medium- and high-frequency range $(1-50 \mathrm{MHz})$ between 1 and $30 \mathrm{~s}$ of averaging time. Such oscillators are also the most stable macroscopic mechanical harmonic oscillators, with fractional frequency stabilities as low as $2.5 \times 10^{-14}$ [54] for room-temperature devices. Over the past decade there has been no major improvement in quartz oscillator performance at room temperature, mainly due to the quartz resonator self-noise. For this reason, the electrodeless [or boîtier à vieillissement amélioré, enclosure with improved aging (BVA)] [55]

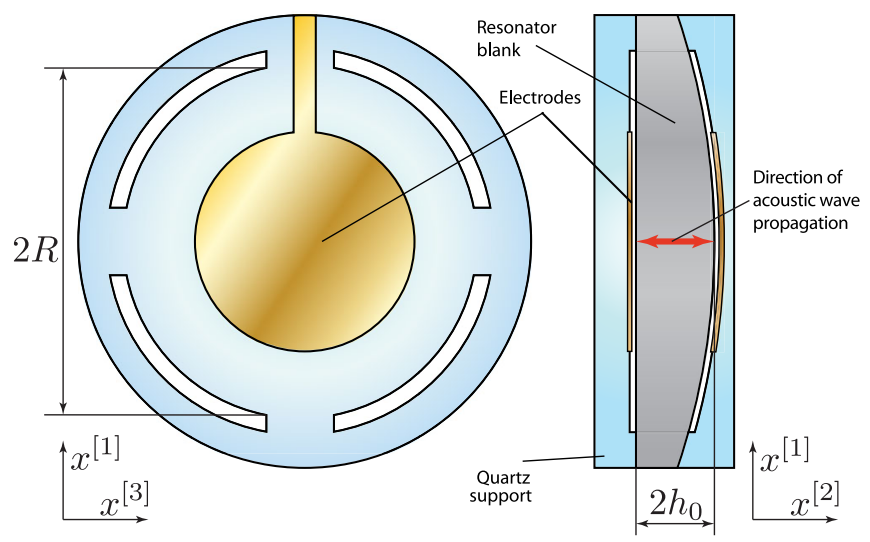

FIG. 5. Schematic view of a BVA (boîtier à vieillissement amélioré, enclosure with improved aging) BAW quartz resonator. The vibrating quartz body, resonator blank, is held by a quartz support deposited electrode. The resonator is fabricated in a plano-convex geometry that traps vibration in the disk center. Electrodes are deposited on the support and separated from the plank by a small vacuum gap. 
BAW quartz resonators (see Fig. 5) have been investigated for cryogenic operation. These investigations reveal extremely high values of the quality factors exceeding $10^{9}$ [56,57], as well as an ability to operate at high overtones [58], providing a new platform for many physical experiments $[59,60]$, for example, detection of high-frequency gravitational waves [61] and cooling a macroscopic object to its ground state for tests of fundamental physics $[58,62]$. Table IV gives values of quality factors for some overtones measured in a $4 \mathrm{~K}$ environment.

Such a significant increase of the quality factor may result in reduction of the oscillator fractional frequency stability. Assuming that the dominant flicker noise of the resonator at $1 \mathrm{~Hz}$ from the carrier $S_{\phi}(1 \mathrm{~Hz}) \sim$ $-130(\mathrm{dBc} / \mathrm{Hz})$ does not change between cryogenic and room temperatures, the Allan deviation of a cryogenic source may be estimated to achieve a level of

$$
\sigma_{y}=\frac{1}{2 Q} \sqrt{2 \ln 2 S_{\phi}(1 \mathrm{~Hz})} \sim 2 \times 10^{-16} .
$$

BAW resonator aging is a systematic drift of its resonant frequency that can be typically observed at long averaging times. This process usually gives a slope of $\tau^{1}$ (where $\tau$ is the integration time) in the Allan deviation curve dominating over the $\tau^{1 / 2}$ law resulting from thermal fluctuations for averaging times over $10^{3} \mathrm{~s}$. The aging process can be caused by a number of effects primarily related to manufacturing. This process is the most prominent during the first months of the oscillator continuous operation, which gradually decreases during that time. For the current experiment, the aging is about $2 \times 10^{-10}$ fractional drift per day. For ultrastable oscillators, this can be reduced to $3 \times 10^{-12}$ fractional drift per day or $1 \times 10^{-9}$ fractional drift per year after at least 90 days of continuous operation.

Another source of stability improvement is associated with the relation between the flicker and white noise in typical BAW oscillators. While the white noise is connected to the signal-to-noise ratio and could thus be reduced by increasing the oscillation power, the flicker noise drops with decreasing power. This situation results in a compromise between the midterm stability (flicker noise

TABLE IV. Values of $Q$ factors for some overtones of cryogenic (4K) BAW resonators.

\begin{tabular}{lccccc}
\hline \hline$X_{n, m, p}$ & $f_{r}(\mathrm{MHz})$ & $Q\left(10^{8}\right)$ & $X_{n, m, p}$ & $f_{r}(\mathrm{MHz})$ & $Q\left(10^{7}\right)$ \\
\hline$C_{3,0,0}$ & 4.99 & 0.4 & $A_{23,0,0}$ & 72.22 & 5.01 \\
$C_{5,0,0}$ & 8.39 & 1.1 & $A_{25,0,0}$ & 78.51 & 2.98 \\
$B_{3,0,0}$ & 5.51 & 0.5 & $A_{27,0,0}$ & 84.78 & 41.2 \\
$B_{5,0,0}$ & 8.39 & 0.6 & $A_{33,0,0}$ & 103.6 & 42.3 \\
$B_{5, m, p}$ & 9.15 & 1.2 & $A_{37,0,0}$ & 116.2 & 49.6 \\
$B_{5, m, p}$ & 9.25 & 2.6 & $A_{43,0,0}$ & 135.0 & 35.6 \\
$A_{3,0,0}$ & 9.37 & 0.6 & $A_{45,0,0}$ & 141.2 & 33.5 \\
$A_{5,0,0}$ & 15.97 & 3 & $A_{47,0,0}$ & 147.5 & 20.0 \\
$A_{15,0,0}$ & 47.11 & 19.3 & $A_{55,0,0}$ & 172.6 & 49.6 \\
\hline \hline
\end{tabular}

region) and short-term stability (white noise region). At cryogenic temperatures the white noise is naturally reduced according to the Nyquist relation, thus giving more room for flicker noise improvement by oscillator power reduction. The Nyquist noise limit for BAW resonators has been recently demonstrated at liquid helium temperatures [63] and unequivocally demonstrates the drop in this limit.

Nevertheless, practical realization of such a cryogenic BAW clock is associated with technical difficulties $[64,65]$. Thus far, only moderate long temperature stability improvement has been demonstrated $[65,66]$. The main problem is the absence of a frequency-temperature turnover point giving rise to significant fluctuations. Additionally, the absence of reliable low-temperature components at the medium- and high-frequency range makes the oscillator design a challenging problem. Whereas the first problem may be overcome by a design of a special cut for cryogenic temperatures, the second is solvable by shifting from semiconductor to superconductor technology. Furthermore, for realizing Lorentz violation experiments, which utilize two oscillators, one just needs to match temperature coefficients of two orthogonally orientated resonators, so as to read-out a stable beat frequency, in a similar way to the cryogenic sapphire oscillator tests in the photon sector [5]. This may relax the requirements for a turnover point for these types of measurements.

\section{E. Potential sensitivity}

Given our expected stability of the cryogenic source given in Eq. (12), we can expect a 4 orders of magnitude improvement in sensitivity, compared to the roomtemperature measurement; thus, a cryogenic quartz oscillator experiment should be able to test Lorentz invariance with a sensitivity to fractional frequency changes of $10^{-19}$. We now estimate the limits that can be derived from such a phonon-sector experiment to the coefficients of the fermions and photons in the SME. Optimistically, perhaps the performance can be improved by a further order of magnitude or more.

The estimates are based on the nonrelativistic singleparticle Hamiltonian describing Lorentz violation in the SME; see, e.g., Eq. (4) in Ref. [8]. This Hamiltonian contains constant terms, terms proportional to the spin $\sigma^{j}$, and terms proportional to momentum $p^{j}$. These terms are unmeasurable as they are either constant or average out over a cycle of the acoustic oscillation. The term proportional to $p^{j} \sigma^{k}$ averages out as well, but can perhaps be measured by applying an oscillating magnetic field at the same frequency as the acoustic oscillation, which would periodically polarize the spin and thus cause a nonzero $\left\langle p^{j} \sigma^{k}\right\rangle$. We do not consider this. Measurable signals arise from two terms in the Hamiltonian. The one proportional to $p^{j} p^{k}$ allows measuring the $\tilde{c}$ coefficients, as discussed in detail above. Using spin-polarized materials, the term proportional to $p^{j} p^{k} \sigma^{l}$ can be measurable. 
Table V shows the order of magnitude of sensitivities from a phonon-sector experiment with a frequency sensitivity of $10^{-18}$. For each entry, all other coefficients for Lorentz violation are assumed to vanish. An asterisk highlights coefficients for which the phonon-sector experiment can provide improved bounds, based on comparison with the maximum sensitivities table in the 2015 edition of the Data Tables on Lorentz and CPT violation [34]. Two asterisks highlight entries where the phonon-sector experiment would provide the first bound on a parameter, based on the same comparison.

The $\tilde{b}_{J}$ coefficients enter from the $p^{j} p^{k} \sigma^{l}$ term. For the estimate, we assume that $\langle\sigma\rangle \sim 10 \%$ of all electron spins and $10^{-6}$ of all nuclear spins have been polarized. The $\tilde{b}_{T}$ coefficients enter through the same mechanism, suppressed by a factor of $\beta_{\oplus}$, Earth's orbital velocity. The $\tilde{d}$ coefficients enter through spin polarization, similar to the $\tilde{b}$ 's.

The fact that bindings in crystals are electromagnetic results in an influence of photon-sector coefficients $[7,67,68]$. This is expected to result in leading-order signals in phonon-sector experiments as reflected in the Table V. A detailed calculation would have to be specific for the material used in the experiment. This is beyond the scope of this paper.

Like the photon terms, the coefficients entering the equations of motion of the valence electrons will modify

TABLE V. Potential order-of-magnitude sensitivities from phonon-sector experiments, assuming a $\delta \nu / \nu=10^{-19}$ resolution. Tabulated is $\log _{10}$ of the expected bound in GeV. Limits in parentheses assume a spin-polarized solid. For entries marked with an asterkisk $(*)$, the sensitivity of phonon-sector experiments is equal to or better than the maximal sensitivity listed in the 2015 edition of Ref. [34] for at least one component. For entries highlighted by two asterisks $(* *)$, no limit is available in Ref. [34] on at least one component.

\begin{tabular}{lcccc}
\hline \hline Coefficient & Electron & Proton & Neutron & Photon \\
\hline$\tilde{b}_{J}$ & $(-18)$ & $(-13)$ & $(-13)$ & \\
$\tilde{b}_{T}$ & $(-14)$ & $(-9)^{*}$ & $(-9)$ & \\
$\tilde{c}_{Q}$ & $-18^{*}$ & -18 & $-18^{*}$ & \\
$\tilde{c}_{-}, \tilde{c}_{J}$ & -18 & -18 & -18 & \\
$\tilde{c}_{T J}$ & -14 & -14 & $-14^{*}$ & \\
$\tilde{c}_{T T}$ & -10 & -10 & -10 & \\
$\tilde{d}_{+}, \tilde{d}_{Q}$ & $(-18)$ & $(-13)^{*}$ & $(-13)$ & \\
$\tilde{d}_{-}, \tilde{d}_{J K}$ & $(-18)$ & $(-13)^{* *}$ & $(-13)$ & \\
$\tilde{d}_{J}$ & $(-18)$ & $(-13)^{* *}$ & $(-13)^{* *}$ & \\
$\tilde{H}_{J T}$ & & & & \\
$\tilde{g}_{c}, \tilde{g}_{Q}, \tilde{g}_{-}, \tilde{g}_{T}$ & & & & \\
$\tilde{g}_{T J}, \tilde{g}_{J K}, \tilde{g}_{D J}$ & & & & $-18^{*}$ \\
$\tilde{\kappa}_{e-}$ & & & & $-14^{*}$ \\
$\tilde{\kappa}_{o+}$ & & & & \\
$\tilde{\kappa}_{e+}$ & & & & -10 \\
$\tilde{\kappa}_{o-}$ & & & & \\
$\tilde{\kappa}_{t r}$ & & & & \\
\hline \hline
\end{tabular}

the bindings, which will perhaps provide additional signals for the electron terms of the SME. These signals will be roughly proportional to the relative change in inertial mass and thus given by the $c^{e}$ coefficients. Their effect may potentially be quite strong. An electron coefficient of, e.g., $\tilde{c}_{Q}^{e}=10^{-20} \mathrm{GeV}$ corresponds to $c_{X X}^{e}+c_{Y Y}^{e}-2 c_{Z Z}^{e} \simeq$ $2 \times 10^{-17}$ because $1 / m^{e} \sim 2000 \mathrm{GeV}^{-1}$, and might thus be measurable. As above, the detailed analysis of specific crystals is beyond the scope of this paper.

\section{SUMMARY AND OUTLOOK}

We present a new method for testing Lorentz symmetry, frequency comparisons between quartz crystal oscillators. While their stability today is surpassed by atomic clocks (especially optical clocks), many tests of Lorentz symmetry are not limited by signal to noise, but often by systematic effects from wobble and tilt of the turntable, and the ability to take data over long stretches of time. Quartz oscillators are compact and are simple to apply and to shield from environmental influences. Their low acceleration sensitivity makes them relatively immune to wobble and tilt. Maintenance-free operation allows for long-term data taking, which helps to make up for the reduced stability. As a demonstration, we improve the laboratory limit on the neutron- $c_{Q}$ coefficient by 6 orders of magnitude, surpassing even current astrophysics bounds. Currently, cryogenic oscillators are under development at University of Western Australia and FEMTO-ST in Besancon, France, and promise strong improvements in stability and sensitivity to Lorentz-violating coefficients.

By analogy to photon-sector experiments, we believe our method can be strongly improved. Photon-sector experiments have gained 4 orders of magnitude in sensitivity over the past 12 years, through higher quality factors resonance and cryogenic operation. A cryogenic version of our experiment may increase the quality factor of the resonance about 10000 -fold and may strongly reduce the temperature coefficient of the oscillators. We thus estimate that 3-4 orders of magnitude improvement to $\sim 10^{-18}$ frequency resolution are realistic. This new technology may also lead to milligram-scale mechanical oscillators at the quantum limit and may see a new brand of ultrastable oscillators. At this sensitivity, the experiment will be able to improve the bounds on several fermion- and photon-sector coefficients, including several coefficients that are not bounded today. Likewise, a detailed theoretical analysis of specific materials might reveal large additional sensitivities to the electron $c$ coefficients.

\section{ACKNOWLEDGMENTS}

We thank Justin Brown and Alan Kostelecký for discussions. This work was supported by the David and Lucile Packard foundation, the Australian Research Council Grants No. CE110001013 and No. DP130100205, the 
Austrian Science Fund (FWF), J3680, and was performed under the auspices of the U.S. Department of Energy by Lawrence Livermore National Laboratory under Contract No. DE-AC52-07NA27344.

[1] D. Colladay and V. A. Kostelecký, CPT Violation and the Standard Model, Phys. Rev. D 55, 6760 (1997).

[2] V. A. Kostelecký and M. Mewes, Sensitive Polarimetric Search for Relativity Violations in Gamma-Ray Bursts, Phys. Rev. Lett. 97, 140401 (2006).

[3] V. A. Kostelecký and M. Mewes, Signals for Lorentz Violation in Electrodynamics, Phys. Rev. D 66, 056005 (2002).

[4] S. Herrmann, A. Senger, K. Möhle, M. Nagel, E. V. Kovalchuk, and A. Peters, Rotating Optical Cavity Experiment Testing Lorentz Invariance at the $10^{-17}$ Level, Phys. Rev. D 80, 105011 (2009).

[5] P. L. Stanwix, M. E. Tobar, P. Wolf, M. Susli, C. R. Locke, E. N. Ivanov, J. Winterflood, and F. van Kann, Test of Lorentz Invariance in Electrodynamics Using Rotating Cryogenic Sapphire Microwave Oscillators, Phys. Rev. Lett. 95, 040404 (2005).

[6] Ch. Eisele, A. Yu. Nevsky, and S. Schiller, Laboratory Test of the Isotropy of Light Propagation at the $10^{-17}$ Level, Phys. Rev. Lett. 103, 090401 (2009).

[7] H. Müller, Testing Lorentz Invariance by the Use of Vacuum and Matter Filled Cavity Resonators, Phys. Rev. D 71, 045004 (2005).

[8] V. A. Kostelecký and C. D. Lane, Constraints on Lorentz Violation from Clock-Comparison Experiments, Phys. Rev. D 60, 116010 (1999).

[9] P. Wolf, F. Chapelet, S. Bize, and A. Clairon, Cold Atom Clock Test of Lorentz Invariance in the Matter Sector, Phys. Rev. Lett. 96, 060801 (2006).

[10] V. Flambaum, S. Lambert, and M. Pospelov, Scalar-Tensor Theories with Pseudoscalar Couplings, Phys. Rev. D 80, 105021 (2009).

[11] F. Canè, D. Bear, D. F. Phillips, M. S. Rosen, C. L. Smallwood, R. E. Stoner, R. L. Walsworth, and V. A. Kostelecký, Bound on Lorentz and CPT Violating Boost Effects for the Neutron, Phys. Rev. Lett. 93, 230801 (2004).

[12] D. Bear, R. E. Stoner, R. L. Walsworth, V. A. Kostelecký, and C. D. Lane, Limit on Lorentz and CPT Violation of the Neutron Using a Two-Species Noble-Gas Maser, Phys. Rev. Lett. 85, 5038 (2000).

[13] M. A. Hohensee, N. Leefer, D. Budker, C. Harabati, V. A. Dzuba, and V. V. Flambaum, Limits on Violations of Lorentz. Symmetry and the Einstein Equivalence Principle Using Radio-Frequency Spectroscopy of Atomic Dysprosium, Phys. Rev. Lett. 111, 050401 (2013).

[14] S. K. Peck, D. K. Kim, D. Stein, D. Orbaker, A. Foss, M. T. Hummon, and L. R. Hunter, Limits on Local Lorentz, Invariance in Mercury and Cesium, Phys. Rev. A 86, 012109 (2012).

[15] J. M. Brown, S. J. Smullin, T. W. Kornack, and M. V. Romalis, New Limit on Lorentz- and CPT-Violating Neutron Spin Interactions, Phys. Rev. Lett. 105, 151604 (2010).
[16] C. Gemmel, W. Heil, S. Karpuk, K. Lenz, Yu. Sobolev, K. Tullney, M. Burghoff, W. Kilian, S. Knappe-Grüneberg, W. Müller, A. Schnabel, F. Seifert, L. Trahms, and U. Schmidt, Limit on Lorentz and CPT Violation of the Bound Neutron Using a Pree precession ${ }^{3} \mathrm{He} /{ }^{129} \mathrm{Xe}$ Comagnetometer, Phys. Rev. D 82, 111901 (2010).

[17] B. Altschul, Disentangling Forms of Lorentz Violation with Complementary Clock Comparison Experiments, Phys. Rev. D 79, 061702 (2009).

[18] M. Smiciklas, J. M. Brown, L. W. Cheuk, S. J. Smullin, and M. V. Romalis, New Test of Local Lorentz Invariance Using a ${ }^{21} \mathrm{Ne}-\mathrm{Rb}-\mathrm{K}$ Comagnetometer, Phys. Rev. Lett. 107, 171604 (2011).

[19] I. Altarev et al., Test of Lorentz Invariance with Spin Precession of Ultracold Neutrons, Phys. Rev. Lett. 103, 081602 (2009).

[20] T. Pruttivarasin, M. Ramm, S. G. Porsev, I. I. Tupitsyn, M. S. Safronova, M. A. Hohensee, and H. Haffner, MichelsonMorley Analogue for Electrons Using Trapped Ions to Test Lorentz Symmetry, Nature (London) 517, 592 (2015).

[21] B. R. Heckel, E. G. Adelberger, C. E. Cramer, T. S. Cook, S. Schlamminger, and U. Schmidt, Preferred-Frame and CP-Violation Tests with Polarized Electrons, Phys. Rev. D 78, 092006 (2008).

[22] R. Bluhm, V. A. Kostelecký, C. D. Lane, and N. Russell, Probing Lorentz and CPT Violation with Space-Based Experiments, Phys. Rev. D 68, 125008 (2003).

[23] A. H. Gomes, V. A. Kostelecký, and A. J. Vargas, Laboratory Tests of Lorentz and CPT Symmetry with Muons, Phys. Rev. D 90, 076009 (2014).

[24] V. A. Kostelecký and J. D. Tasson, Matter-Gravity Couplings and Lorentz Violation, Phys. Rev. D 83, 016013 (2011).

[25] I. S. Grudinin, H. Lee, O. Painter, and K. J. Vahala, Phonon Laser Action in a Tunable Two-Level System, Phys. Rev. Lett. 104, 083901 (2010).

[26] X. Luan, Y. Huang, Y. Li, J. F. McMillan, J. Zheng, S.-W. Huang, P.-C. Hsieh, T. Gu, D. Wang, A. Hati, D. A. Howe, G. Wen, M. Yu, G. Lo, D.-L. Kwong, and C. Wei Wong, An Integrated Low Phase Noise Radiation-Pressure-Driven Optomechanical Oscillator Chipset, Sci. Rep. 4, 6842 (2014).

[27] H. Seok, E. M. Wright, and P. Meystre, Dynamic Stabilization of an Optomechanical Oscillator, Phys. Rev. A 90, 043840 (2014).

[28] M. Hossein-Zadeh and K. J. Vahala, Observation of Injection Locking in an Optomechanical rf Oscillator, Appl. Phys. Lett. 93, 191115 (2008).

[29] J. Li, X. Yi, H. Lee, S. A. Diddams, and K. J. Vahala, Electro-optical Frequency Division and Stable Microwave Synthesis, Science 345, 309 (2014).

[30] M. Tomes and T. Carmon, Photonic Micro-Electromechanical Systems Vibrating at X-Band $(11-G H z)$ Rates, Phys. Rev. Lett. 102, 113601 (2009).

[31] I. S. Grudinin, A. B. Matsko, and L. Maleki, Brillouin Lasing with a $\mathrm{CaF}_{2}$ Whispering Gallery Mode Resonator, Phys. Rev. Lett. 102, 043902 (2009).

[32] H. Lee, T. Chen, J. Li, K Y. Yang, S. Jeon, O. Painter, and K. J. Vahala, Chemically Etched Ultrahigh-Q WedgeResonator on a Silicon Chip, Nat. Photonics 6, 369 (2012). 
[33] J. Li, H. Lee, T. Chen, and K. J. Vahala, Characterization of a High Coherence, Brillouin Microcavity Laser on Silicon, Opt. Express 20, 20170 (2012).

[34] V. A. Kostelecký and N. Russell, Data Tables for Lorentz. and CPT Violation, Rev. Mod. Phys. 83, 11 (2011).

[35] A. P. Lightman and D. L. Lee, Restricted Proof That the Weak Equivalence Principle Implies the Einstein Equivalence Principle, Phys. Rev. D 8, 364 (1973).

[36] H. B. Nielsen and I. Picek, Lorentz Non-Invariance, Nucl. Phys. B211, 269 (1983).

[37] C. M. Will, Theory and Experiment in Gravitational Physics (Cambridge University Press, Cambridge, England, 1993).

[38] S. Coleman and S. L. Glashow, High-Energy Tests of Lorentz Invariance, Phys. Rev. D 59, 116008 (1999).

[39] V. A. Kostelecký and M. Mewes, Constraints on Relativity Violations from Gamma-Ray Bursts, Phys. Rev. Lett. 110, 201601 (2013).

[40] A. A. Michelson and E. W. Morley, On the Relative Motion of the Earth and the Luminiferous Ether, Am. J. Sci. 34, 333 (1887); On the Relative Motion of the Earth and the Luminiferous Aether, Philos. Mag. 24, 449 (1887); R. J. Kennedy and E. M. Thorndike, Experimental Establishment of the Relativity of Time, Phys. Rev. 42, 400 (1932); H. E. Ives and G. R. Stilwell, An Experimental Study of the Rate of a Moving Atomic Clock, J. Opt. Soc. Am. 28, 215 (1938).

[41] M. Hohensee, A. Glenday, C.-H. Li, M. E. Tobar, and P. Wolf, Erratum: New Methods of Testing Lorentz Violation in Electrodynamics, Phys. Rev. D 75, 049902(E) (2007); P. L. Stanwix, M. E. Tobar, P. Wolf, C. R. Locke, and E. N. Ivanov, Improved Test of Lorentz Invariance in Electrodynamics Using Rotating Cryogenic Sapphire Oscillators, Phys. Rev. D 74, 081101 (2006); P. Antonini, M. Okhapkin, E. Göklü, and S. Schiller, Reply to "Comment on 'Test of Constancy of Speed of Light with Rotating Cryogenic Optical Resonators,', Phys. Rev. A 72, 066102 (2005); M. E. Tobar, P. Wolf, and P. L. Stanwix, Comment on "Test of Constancy of Speed of Light with Rotating Cryogenic Optical Resonators," Phys. Rev. A 72, 066101 (2005); S. Herrmann, A. Senger, E. Kovalchuk, H. Müller, and A. Peters, Test of the Isotropy of the Speed of Light Using a Continuously Rotating Optical Resonator, Phys. Rev. Lett. 95, 150401 (2005); J. Ehlers, C. Lämmerzahl, M. E. Tobar, P. L. Stanwix, M. Susli, P. Wolf, C. R. Locke, and E. N. Ivanov, in Special Relativity, Lecture Notes in Physics, Vol. 702 (Springer, Berlin, 2006) pp. 416; P. L. Stanwix, M. E. Tobar, P. Wolf, M. Susli, C. R. Locke, E. N. Ivanov, J. Winterflood, and F. van Kann, Test of Lorentz Invariance in Electrodynamics Using Rotating Cryogenic Sapphire Microwave Oscillators, Phys. Rev. Lett. 95, 040404 (2005); P. Antonini, M. Okhapkin, E. Göklü, and S. Schiller, Test of Constancy of Speed of Light with Rotating Cryogenic Optical Resonators, Phys. Rev. A 71, 050101 (2005); M. E. Tobar, P. Wolf, A. Fowler, and J. G. Hartnett, New Methods of Testing Lorentz Violation in Electrodynamics, Phys. Rev. D 71, 025004 (2005); P. Wolf, S. Bize, A. Clairon, G. Santarelli, M. E. Tobar, and A. N. Luiten, Improved Test of Lorentz Invariance in Electrodynamics, Phys. Rev. D 70 , 051902 (2004); H. Müller, S. Herrmann, A. Saenz, A. Peters, and C. Lämmerzahl, Optical Cavity Tests of Lorentz Invariance for the Electron, Phys. Rev. D 68, 116006
(2003); H. Müller, S. Herrmann, C. Braxmaier, S. Schiller, and A. Peters, Modern Michelson-Morley Experiment Using Cryogenic Optical Resonators, Phys. Rev. Lett. 91, 020401 (2003); J. A. Lipa, J. A. Nissen, S. Wang, D. A. Stricker, and D. Avaloff, New Limit on Signals of Lorentz Violation in Electrodynamics, Phys. Rev. Lett. 90, 060403 (2003).

[42] M. Nagel, S. R. Parker, E. V. Kovalchuk, P. L. Stanwix, J. G. Hartnett, E. N. Ivanov, A. Peters, and M. E. Tobar, Direct Terrestrial Test of Lorentz. Symmetry in Electrodynamics to $10^{-18}$, Nat. Commun. 6, 8174 (2015).

[43] M. A. Hohensee, P. L. Stanwix, M. E. Tobar, S. R. Parker, D. F. Phillips, and R. L. Walsworth, Improved Constraints on Isotropic Shift and Anisotropies of the Speed of Light Using Rotating Cryogenic Sapphire Oscillators, Phys. Rev. D 82, 076001 (2010).

[44] F. N. Baynes, M. E. Tobar, and A. N. Luiten, Oscillating Test of the Isotropic Shift of the Speed of Light, Phys. Rev. Lett. 108, 260801 (2012).

[45] IEEE Standard on Piezoelectricity, ANSI/IEEE Std 1761987, O_1, 1988, http://ieeexplore.ieee.org/xpls/abs_all.jsp? arnumber $=26560$.

[46] R. L. Filler, The Acceleration Sensitivity of Quartz Crystal Oscillators: A Review, IEEE Trans. Ultrason. Ferroelectr. Freq. Control 35, 297 (1988).

[47] D. S. Stevens and H. F. Tiersten, An Analysis of Doubly Rotated Quartz Resonators Utilizing Essentially Thickness Modes with Transverse Variation, J. Acoust. Soc. Am. 79, 1811 (1986).

[48] E. P. EerNisse, Analysis of Thickness Modes of Contoured, Doubly Rotated, Quartz Resonators, IEEE Trans. Ultrason. Ferroelectr. Freq. Control 48, 1351 (2001).

[49] J. D. Prestage, J. J. Bollinger, W. M. Itano, and D. J. Wineland, Limits for Spatial Anisotropy by Use of Nuclear-Spin-Polarized ${ }^{9} \mathrm{Be}^{+}$Ions, Phys. Rev. Lett. 54, 2387 (1985).

[50] S. K. Lamoreaux, J. P. Jacobs, B. R. Heckel, F. J. Raab, and E. N. Fortson, New Limits on Spatial Anisotropy from Optically Pumped ${ }^{201} \mathrm{Hg}$ and ${ }^{199} \mathrm{Hg}$, Phys. Rev. Lett. 57, 3125 (1986).

[51] T. E. Chupp, R. J. Hoare, R. A. Loveman, E. R. Oteiza, J. M. Richardson, M. E. Wagshul, and A. K. Thompson, Results of a New Test of Local Lorentz. Invariance: A Search for Mass Anisotropy in ${ }^{21} \mathrm{Ne}$, Phys. Rev. Lett. 63, 1541 (1989).

[52] B. Altschul, Limits on Neutron Lorentz Violation from the Stability of Primary Cosmic Ray Protons, Phys. Rev. D 78, 085018 (2008).

[53] M. A. Hohensee, S. Chu, A. Peters, and H. Müller, Equivalence Principle and Gravitational Redshift, Phys. Rev. Lett. 106, 151102 (2011).

[54] P. Salzenstein, A. Kuna, L. Sojdr, and J. Chauvin, Significant Step in Ultra-High Stability Quartz Crystal Oscillators, Electron. Lett. 46, 1433 (2010).

[55] R. J. Besson, A New "Electrodeless" Resonator Design, in Proceedings of the 31st Annual Symposium on Frequency Control, 1977 (IEEE, Atlantic City, New Jersey, 1977), pp. 147-152.

[56] S. Galliou, J. Imbaud, M. Goryachev, R. Bourquin, and P. Abbe, Losses in High Quality Quartz Crystal Resonators at Cryogenic Temperatures, Appl. Phys. Lett. 98, 091911 (2011). 
[57] M. Goryachev, D. L. Creedon, E. N. Ivanov, S. Galliou, R. Bourquin, and M. E. Tobar, Extremely Low-Loss Acoustic Phonons in a Quartz Bulk Acoustic Wave Resonator at Millikelvin Temperature, Appl. Phys. Lett. 100, 243504 (2012).

[58] M. Goryachev, D. L. Creedon, S. Galliou, and M. E. Tobar, Observation of Rayleigh Phonon Scattering through Excitation of Extremely High Overtones in Low-Loss Cryogenic Acoustic Cavities for Hybrid Quantum Systems, Phys. Rev. Lett. 111, 085502 (2013).

[59] S. Galliou, M. Goryachev, R. Bourquin, P. Abbe, J. P. Aubry, and M.E. Tobar, Extremely Low Loss PhononTrapping Cryogenic Acoustic Cavities for Future Physical Experiments, Sci. Rep. 3, 2132 (2013).

[60] M. Goryachev and M. E. Tobar, Effects of Geometry on Quantum Fluctuations of Phonon-Trapping Acoustic Cavities, New J. Phys. 16, 083007 (2014).

[61] M. Goryachev and M.E. Tobar, Gravitational Wave Detection with High Frequency Phonon Trapping Acoustic Cavities, Phys. Rev. D 90, 102005 (2014).

[62] I. Pikovski, M. R. Vanner, M. Aspelmeyer, M. S. Kim, and C. Brukner, Probing Planck-Scale Physics with Quantum Optics, Nat. Phys. 8, 393 (2012).
[63] M. Goryachev, E. N. Ivanov, F. van Kann, S. Galliou, and M. E. Tobar, Observation of the Fundamental Nyquist Noise Limit in an Ultra-High q-Factor Cryogenic Bulk Acoustic Wave Cavity, Appl. Phys. Lett. 105, 153505 (2014).

[64] M. Goryachev, S. Galliou, P. Abbe, P. Bourgeois, S. Grop, and B. Dubois, Quartz Resonator Instabilities under Cryogenic Conditions, IEEE Trans. Ultrason. Ferroelectr. Freq. Control 59, 21 (2012).

[65] M. Goryachev, S. Galliou, J. Imbaud, and P. Abbé, Advances in Development of Quartz Crystal Oscillators at Liquid Helium Temperatures, Cryogenics 57, 104 (2013).

[66] M. Goryachev, S. Galliou, J. Imbaud, R. Bourquin, and Ph. Abbé, in Proceedings of the European Frequency and Time Forum and IEEE International Frequency Control Symposium Joint Meeting, San Francisco, 2011 (IEEE, San Francisco, 2011).

[67] H. Müller, S. Herrmann, A. Saenz, A. Peters, and C. Lämmerzahl, Optical Cavity Tests of Lorentz Invariance for the Electron, Phys. Rev. D 68, 116006 (2003).

[68] H. Müller, S. Herrmann, A. Saenz, A. Peters, and C. Lämmerzahl, Tests of Lorentz Invariance Using Hydrogen Molecules, Phys. Rev. D 70, 076004 (2004). 